\title{
Satisfacción de usuarios de los servicios de salud: Factores sociodemográficos y de accesibilidad asociados. Perú, 2000
}

\author{
Juan Seclén-Palacin ${ }^{1}$, Christian Darras ${ }^{2}$
}

Resumen

Objetivos: Determinar el nivel de satisfacción del usuario (SU) en centros de salud (CS) y hospitales del MINSA; analizar la relación entre SU y nivel socioeconómico (NSE); e identificar los factores sociodemográficos y de accesibilidad asociados. Materiales y Métodos: Análisis secundario de los datos de Encuesta Nacional de Niveles de Vida (ENNIV) 2000. Fueron entrevistados 376 y 327 usuarios de los CS y hospitales, respectivamente. Las variables de estudio fueron $S U$, niveles socioeconómico y características sociodemográficas y accesibilidad para la atención. Se aplicó estadística descriptiva y multivariada. Resultados: Los usuarios de los establecimientos muestran características diferenciadas. La SU fue 68,1\% y 62,1\% para los CS y hospitales, respectivamente. Los usuarios de menor NSE presentaron mayor satisfacción. La edad, educación, distancia al establecimiento y el tiempo de espera presentan asociaciones con la SU. Conclusiones: Existen factores sociodemográficos, económicos y de accesibilidad para la atención, que muestran relación con la satisfacción del usuario; tales factores se comportan de manera particular según el tipo de establecimiento de salud (centro de salud, hospital), debido fundamentalmente a que la población usuaria es distinta. Esto último relativiza la comparación del nivel de satisfacción y de sus factores asociados entre tales establecimientos.

Palabras clave Servicios de salud; satisfacción de los consumidores de servicios de salud; satisfacción del paciente; calidad de la atención en salud.

Peruvian health services users' satisfaction: Socio-demographic factors and accessibility. Peru 2000

\section{Abstract}

Objectives: To determine users' satisfaction in Peru Ministry of Health's health centers (HC) and hospitals, to analyze the relation between users' satisfaction and the socioeconomic variable, and to identify both the sociodemographic and accessibility factors related. Materials

\footnotetext{
1 Médico, postgrado en epidemiología y maestro en gobierno y gerencia en salud. Consultor externo OPS, representación Perú. Profesor investigador asociado, Facultad de Salud Pública y Administración, Universidad Peruana Cayetano Heredia. Lima, Perú.

2 Médico, Master en Salud Pública. Consultor en sistemas y servicios de salud. Representante de la Organización Panamericana de la Salud en Bolivia.
}

and Methods: Secondary analysis of the database of Peruvian Living Standards Survey (PLSS), year 2000; 376 health center and 327 hospital users were studied. The study variables were users'satisfaction, socioeconomic level, socio-demographic variables and accessibility to care. Descriptive and multivariate statistics were applied. Results: The health center users show patterns different to the hospital users. Satisfaction was respectively $68,1 \%$ and $62,1 \%$ for health centers and hospitals. The users with less socioeconomic level showed greater satisfaction. Age, education, distance to health facilities and waiting time were related with users'satisfaction. Conclusions: Socio-demographic, economic and accessibility to care factors associated to users' satisfaction show specific characteristics according to location, probably due to difference between health facilities populations.

Keywords: Health services; consumer satisfaction; patient satisfaction; quality of health care. 


\section{INTRODUCCIÓN}

La satisfacción del usuario (SU) es uno de los aspectos que en términos de evaluación de los servicios de salud y calidad de atención, ha venido cobrando mayor atención en salud pública ( $\left.{ }^{1}\right)$ siendo considerada desde hace poco más de una década uno de los ejes de evaluación de servicios de salud $\left({ }^{2}\right)$. Si bien es cierto existe un intenso debate en cuanto a su concepción y metodologías de medición, también es consenso la relevancia de la visión de los usuarios sobre los servicios como un elemento clave en la mejoría de la organización y provisión de los servicios de salud ${ }^{(3,4)}$.

El tema de SU es todo un desafío a la salud pública y los gestores y planificadores en salud, los dedicados a la academia, investigadores y usuarios en general. No existe un consenso claro sobre su significado y concepción pero es evidente que refleja una visión diferente y estratégica de los servicios de salud. La definición de Pascoe: "comparación de la experiencia del paciente con sus estándares subjetivos al salir de la atención sanitaria" es una de las mas simples y aceptadas $\left({ }^{5}\right)$. La SU es considerada como una respuesta actitudinal y de juicio de valor que el usuario construye producto de su encuentro e interacción con el servicio.

La SU es apreciada como relevante al menos en 4 áreas: comparar sistemas o programas de salud, evaluar la calidad de los servicios de salud $\left(^{6}\right)$, identificar cuáles son los aspectos de los servicios que necesitan de cambio para mejorar la satisfacción y asistir a las organizaciones sociales en la identificación de consumidores con poca aceptabilidad de los servicios $\left({ }^{7}\right)$. También se relaciona con la conducta del paciente y sus resultados en términos de salud individual.

Desde mediados de la década pasada, algunos autores han expresado que las encuestas de SU generalmente muestran alta satisfacción y poca variabilidad, lo que dificulta su uso en términos de gestión; sin embargo, otros estudios señalan que la introducción de preguntas que exploren y caractericen las experiencias de los pacientes con aspectos específicos de la atención son más informativas y útiles $\left({ }^{8}\right)$. Adicionalmente, la complementariedad con técnicas de investigación cualitativa fortalecen la riqueza de la información y su potencial uso en la gestión. Como mencionamos anteriormente, la satisfacción es un fenómeno básicamente psicológico que requiere de metodologías cualitativas para complementar su estudio.
Según algunos estudios, los niveles de $\mathrm{SU}$ en atenciones ambulatorias oscilan entre 60 y $70 \%$, observándose especificidades en función al público estudiado, servicio y/o establecimiento proveedor de la atención, motivo de consulta, momento de entrevista y método utilizado $\left({ }^{9,10}\right)$. Reportes internacionales han evidenciado altos índices de SU en establecimientos de salud públicos y adicionalmente su poca variabilidad durante los estudios de seguimiento, lo cual ha despertado algunos cuestionamientos en términos de su utilidad como instrumento de monitoreo y valor en la gerencia de servicios $\left({ }^{11}\right)$. En sistemas de salud de países desarrollados (Reino Unido, Canadá, EEUU, España) y en algunos en vías de desarrollo (Brasil, México, Chile, Nueva Zelanda, entre otros) existen políticas sanitarias que impulsan el desarrollo de todo un sistema de información gerencial que se inicia con el recojo periódico de opiniones de los usuarios sobre los servicios de salud, continuando con el procesamiento y generación de información puesta a disposición de los diversos decisores sanitarios, con la finalidad de inducir acciones de mejoría en sus servicios. En tales países, esta concepción traducida en política y acción representa un eje importante en gestión sanitaria. Jackson y colaboradores consideran que el valor de la SU depende del objetivo para el cual se mide la satisfacción $\left({ }^{12}\right)$. Nosotros consideramos que si la idea es disponer de información para comparar los resultados de ajustes organizacionales o iniciativas de mejoría de calidad desarrolladas por servicios / establecimientos de salud, sería recomendable la medición de la satisfacción global con la atención (satisfacción con "enfoque resultado"). Por el contrario, si el propósito de medir SU es la identificación de aspectos organizacionales y del proceso de atención que precisan ser mejorados en un servicio de salud, el estudio debería enfocarse principalmente a los componentes de dicha atención (satisfacción con "enfoque proceso"). Para cada uno de esos enfoques existe un conjunto de herramientas útiles y validadas; además. estos enfoques no son, en lo absoluto, excluyentes ente sí.

Según la información acumulada, existen varios factores que influyen en la satisfacción de los usuarios de los servicios de salud. Sistematizándolos podemos agruparlos en: a) factores individuales: demográficos (edad, sexo, raza), sociales (estado marital, grupo social, nivel educacional, dinámica organizacional de la comunidad, redes de participación social), económicos (nivel de ingresos, gastos), culturales (etnia, expectativas $\left({ }^{13}\right)$, concepciones culturales sobre los servicios de salud y el proceso salud-riesgo-enfermedad), experiencia con el 
servicio (desenlace del contacto previo entre el usuario y el servicio); b) factores familiares / sociales: experiencias con el servicio de parte de amigos, familiares, de la red social circundante, conducta y concepción del entorno familiar sobre el proceso salud-enfermedad; y, c) factores del servicio de salud: de accesibilidad geográfica (distancia al servicio de salud) y otros dependientes de la propia organización del servicio de salud (comunicación interpersonal, resolutividad del servicio, tiempo de espera para la atención, eficacia de las acciones, trato y cordialidad del personal, disponibilidad de medicamentos, confórt del local, privacidad, ente otros) $\left({ }^{14,15,16,17,18}\right)$. La interacción entre estos factores resultan en el nivel de aceptabilidad y satisfacción; dicha interacción es compleja, ya que tratamos de un aspecto de base subjetiva, es decir, basados en percepciones y experiencias (sustento psicológico).

En el Perú, desde mediados de los años 90, se han desarrollado algunas iniciativas puntuales y de corto alcance en el tema de escucha al usuario, impulsados fundamentalmente por algunos proyectos de cooperación internacional (por ejemplo, proyecto $2000\left({ }^{19}\right)$, salud y nutrición básica, cobertura con calidad, entre otros), que consideraron dentro de su marco de referencia teóricooperacional la mejoría continua de la calidad y la satisfacción. Desafortunadamente, tales iniciativas tuvieron un escenario de aplicación bastante reducido y limitaciones en su institucionalización. Desde esa misma época, el Ministerio de Salud ha formulado algunos lineamientos en salud dirigidos al usuario; empero su cristalización como praxis regular, permanente e inmersa en el accionar de los gestores y proveedores de atención continúa siendo un gran desafío $\left.{ }^{(20,21}\right)$. Asimismo, la disponibilidad de información sobre el nivel de SU de los servicios de salud públicos (hospitales y centros de salud) es limitada y no se conoce de estudios basados en muestras nacionales que exploren las características de SU y sus diferencias en función de factores sociales, económicos, demográficos, culturales y de las características de la oferta. Dentro de las políticas públicas actuales de reducción de las desigualdades e inequidad en salud ${ }^{(22}$, se plantea el desafío de explorar la asociación del nivel de SU según condición económica. La literatura muestra poca información sobre el particular y la existente aun no es concluyente. Es en ese aspecto que el presente estudio pretende contribuir.

Fueron objetivos del estudio analizar el nivel de satisfacción de los usuarios de establecimientos del MINSA del Perú, su relación con el nivel socioeconómico e identificar los principales factores asociados de tipo social, demográfico, y de accesibilidad a la atención, referente al año 2000.

\section{MATERIALES Y MÉTODOS}

Se realizó un análisis de datos secundarios de la Encuesta Nacional de Niveles de Vida realizada en el año 2000 (ENNIV 2000). Dicha encuesta fue transversal, con representatividad nacional, siendo su muestreo probabilístico, multietápico y proporcional a las regiones del Perú. Para cada una de las regiones estudiadas se seleccionó un mínimo muestral, visitándose en total 3997 hogares, obteniendo datos de 19957 personas. Las viviendas seleccionadas fueron visitadas por los encuestadores de campo, quienes entrevistaron al jefe del hogar para obtener información general. Para el caso de información específica, se entrevistó al resto de miembros del hogar, lo cual motivó realizar varias visitas al domicilio de estudio.

La muestra fue seleccionada de la siguiente forma: del total global de 19957 personas se escogió aquellas mayores de 16 años, 12490 (62,6\%); de este subgrupo, se eligió las que autorreportaron enfermedad, malestar o accidente (EMA) en las últimas 4 semanas $(n=3176$ personas, $25,4 \%)$. De este conjunto de personas, se volvió a seleccionar aquellas que accedieron a algún tipo de consulta $(\mathrm{n}=1608,50,6 \%)$, de las cuales un total de $703(43,7 \%)$ consultó establecimientos de salud del MINSA (centros de salud y hospitales). Este último subgrupo fue objeto de análisis del presente estudio. Las subpoblaciones de análisis se las muestra resumidamente en la Figura 1. La unidad de análisis del presente estudio fueron los usuarios de los centros de salud y hospitales del MINSA.

Las variables fueron satisfacción del usuario con la atención (definida como la percepción del entrevistado que expresa su juicio de valor con la atención recibida en el establecimiento. Siendo sus valores satisfactoria $=2$, poco satisfactoria $=1$, e insatisfecho $=0$ ) $\mathrm{y}$ establecimiento de salud (lugar donde el usuario recibió la atención: centro de salud y hospital). El nivel socioeconómico fue definido utilizando la metodología de línea de pobreza, la cual se basa en un conjunto de canastas que contienen bienes y servicios de acuerdo a las regiones geográficas del país. Estas canastas consideran una provisión equilibrada y adecuada de nutrientes para los miembros del hogar. Asimismo, éstas han sido valorizadas a precios de cada uno de los dominios de estudio de la ENNIV 2000, con el fin de evitar el sesgo que pudiera 


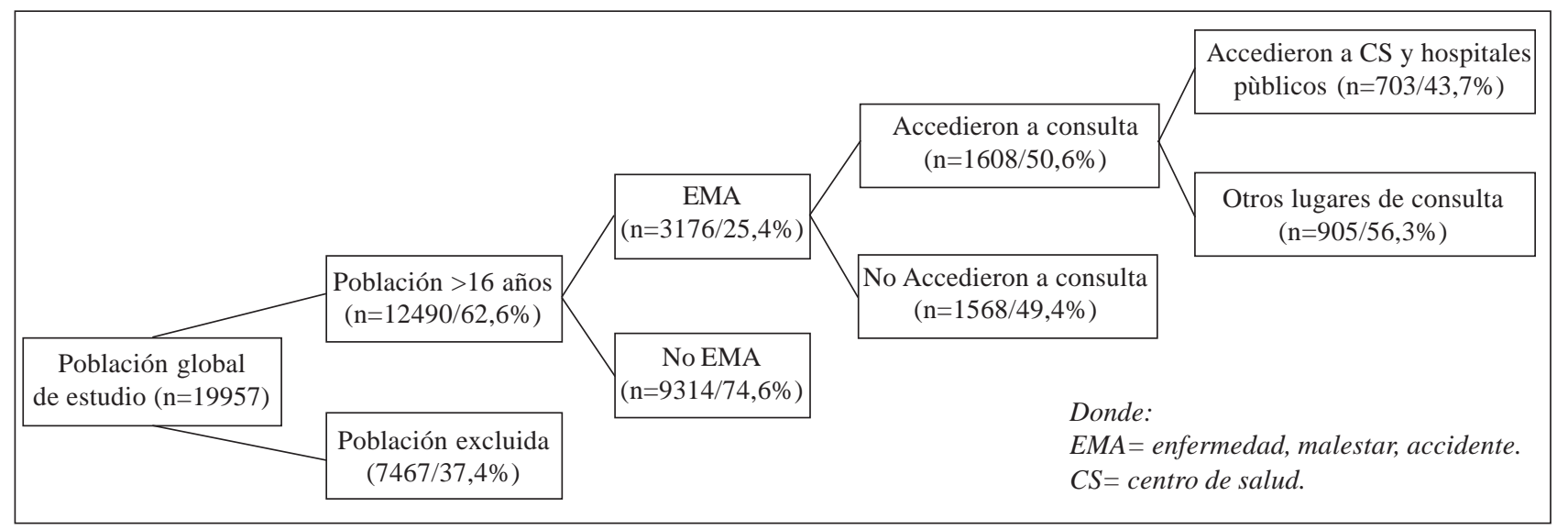

Figura 1. Selección de la población para el presente estudio (ENNIV 2000).

introducirse al utilizar canastas de consumo sólo a precios urbanos. Se utilizaron dos líneas de pobreza. Se encontraban en situación de pobreza extrema aquellos hogares cuyo gasto total per cápita era menor que el costo de una canasta básica alimentaria (CBA), que cubre requerimientos mínimos nutricionales. Fueron considerados pobres los hogares cuyo gasto per cápita fue menor que el costo de una canasta básica de consumo (CBC), la cual es igual al valor de la CBA más el costo de otros bienes y servicios no alimentarios. El valor de la CBC se ha obtenido multiplicando el valor de la CBA por el inverso del coeficiente que mide la proporción del gasto en alimentos respecto al gasto total. El valor del coeficiente se ha obtenido en el decil que tiene un nivel de gasto en alimentos similar al valor de la canasta alimentaria. Adicionalmente, se consideró el nivel educacional (último nivel de estudio aprobado por el entrevistado, siendo categorizado en nivel primario, secundaria, superior y ninguno), lengua materna (lengua hablada de la madre del entrevistado, pudiendo ser español y nativa - quechua, aymara, shipibo u otros-) y sociodemográficas, como edad, sexo y estado civil. Finalmente, fue considerada como medición del acceso geográfico la variable distancia al establecimiento (tiempo en minutos que el entrevistado demoró para llegar al establecimiento de salud donde recibió la atención), y como medición del acceso a la atención se consideró tiempo de espera para la atención (tiempo en minutos desde la llegada del usuario al establecimiento y su respectiva entrada al consultorio).

Con fines de identificar los factores asociados a satisfacción fue creada una variable síntesis, dependiente y binaria llamada satisfacción del usuario, la cual resultó de la recategorización de la variable primitiva satisfacción con la atención recibida, siendo la categoría satisfactoria recatalogada como "usuario satisfecho", mientras que las categorías "poco satisfactoria" y "no le satisfizo" fueron recodificadas como "usuario insatisfecho".

El cuestionario ENNIV es utilizado rutinariamente en nuestro país desde inicios de la década de los '90. Está compuesto por secciones generales del hogar y otras específicas a los individuos; estas últimas incluyen preguntas del campo educacional, económico, social, laboral, productivo, cultural y de salud. El área de salud incluye dos grupos de preguntas: una de ellos dirigido a todos los miembros del hogar y el restante sólo aplicable a las mujeres en edad fértil. Para este estudio trabajaremos con los datos provenientes del primer grupo. La parte del cuestionario de utilidad para este análisis incluye preguntas de autopercepción del estado de salud ("Durante las últimas 4 semanas, ¿usted estuvo enfermo o accidentado, tuvo algún síntoma o malestar?). Asimismo, pregunta sobre el acceso a la atención (" ¿tuvo alguna consulta de salud en las últimas 4 semanas para atenderse por esa enfermedad o accidente?”), lugar de atención (“¿dónde se llevó a cabo la consulta?”). La pregunta índice tiene relación con la variable satisfacción con la atención recibida $\{$ ¿Considera que la atención recibida por el "médico, enfermera", en el "centro de salud, hospital" ha sido "satisfactoria", poco satisfactoria», "no le satisfizo?". Esta pregunta sólo fue formulada según el diseño del estudio a las personas que reportaron acceder a consulta por motivo de enfermedad, accidente, malestar en las últimas 4 semanas. Adicionalmente, se aplicó un grupo de preguntas relacionadas con las variables sociodemográficas y de 
accesibilidad para la atención. El trabajo de campo fue realizado vía visitas domiciliarias, por entrevistadores altamente entrenados en el uso del instrumento, siendo el jefe del hogar y los miembros de las familias quienes respondieron a las preguntas del cuestionario.

En las técnicas de procesamiento y análisis de datos, se utilizó análisis de estadística descriptiva (frecuencia, proporciones), análisis bivariado para estudiar la relación entre la satisfacción y el nivel económico en centros de salud y hospitales, análisis multivariado (regresión logística múltiple) para determinar las variables asociadas al nivel de satisfacción. La ecuación considerada fue:

Satisfacción del usuario con la atención = fx (edad, sexo, educación, estado civil, lengua materna), (nivel económico), (accesibilidad geográfica), (accesibilidad para la atención) + error.

El análisis de residuales fue realizado con el fin de hacer los ajustes estadísticos y estudiar la normalidad del modelo. Análisis de colinearidad se realizó para identificar variables independientes con alta nivel de correlación ( $r>0,8$ significa exclusión del modelo). Se hizo también análisis de interacción, sobre todo para aquellas variables que mostraron niveles próximos a la significancia. Las variables consideradas como significantes fueron aquellas con un odds ratio (OR) mayor o igual de 1,5 con un nivel de significancia mínimo $p=0,05$, valores de significancia aceptados si el valor $p$ fue $<0,05$. La base de datos ENNIV 2000 fue puesta a disposición de los autores por la Organización Panamericana de la Salud (Representación Perú). En relación al control de calidad, se realizó análisis de consistencia, verificando la distribución de valores para cada una de las variables a estudiar. Caso de valores inconsistentes se consultó con personal del Instituto Cuánto (agencia que realizó el estudio ENNIV), a fin de recibir sugerencias.

\section{RESULTADOS}

Con relación a las características socio-demográficas y económicas de la población de estudio (Tabla 1), la edad del grupo que asistió a hospitales y centros de salud MINSA muestra particularidades, así en los CS los menores de 29 años constituyeron el $31 \%$, los de 30 a 49 años $33,5 \%$ y los mayores de 50 años $35,4 \%$; en los hospitales, 24,2\% de los atendidos fue mayor de 60 años, mientras que en los centros de salud dicha población alcanzó $18,1 \%$. Por otro lado, la población adolescente (16 a 19 años) atendida en los CS fue $12 \%$, mientras que en hospitales fue $8,6 \%$. Estas variaciones de edad para las poblaciones de CS y hospitales fueron significativas, sobre todo para los extremos de edad (adolescentes y ancianos). Existe una tendencia de la población atendida en los CS a ser más joven en oposición a los hospitales, donde la población es un tanto mayor (media de la población CS $=40,4$ años, media en hospitales $=44,4$ años, prueba " $\mathrm{t} "=-2,544, p=0,013)$.

Con relación al sexo, existió una muy ligera predominancia de la población femenina en hospitales y CS $(57,7$ y $58,7 \%$, respectivamente).

Tabla 1. Caracterización socio-demográfica de la población que consultó en establecimientos de salud del MINSA. ENNIV 2000 ( $n=703)$.

\begin{tabular}{|c|c|c|c|}
\hline $\begin{array}{l}\text { Factores } \\
\text { sociodemo- } \\
\text { gráficos }\end{array}$ & $\begin{array}{c}\text { Centros } \\
\text { de salud } \\
\text { n (\%) }\end{array}$ & $\begin{array}{c}\text { Hospitales } \\
\text { n (\%) }\end{array}$ & $\begin{array}{l}\text { Total } \\
\text { n (\%) }\end{array}$ \\
\hline \multicolumn{4}{|c|}{ Grupos etáreos (años): } \\
\hline $16-19$ & $45(12,0)$ & $28(8,6)$ & $73(10,4)$ \\
\hline $20-29$ & $79(21,0)$ & $66(20,2)$ & $145(20,6)$ \\
\hline $30-39$ & $68(18,1)$ & $51(15,6)$ & $119(16,9)$ \\
\hline $40-49$ & $61(16,2)$ & $56(17,1)$ & $117(16,6)$ \\
\hline $50-59$ & $55(14,6)$ & $47(14,4)$ & $102(14,5)$ \\
\hline$>60$ & $68(18,1)$ & $79(24,2)$ & $147(20,9)$ \\
\hline \multicolumn{4}{|l|}{ Sexo: } \\
\hline Hombre & $159(42,3)$ & $135(41,3)$ & $284(41,8)$ \\
\hline Mujer & $217(57,7)$ & $192(58,7)$ & $409(58,2)$ \\
\hline \multicolumn{4}{|c|}{ Nivel educacional: } \\
\hline Ninguno & $44(11,7)$ & $27(8,3)$ & $71(10,1)$ \\
\hline Transición & $7(1,9)$ & $4(1,2)$ & $11(1,6)$ \\
\hline Primaria & $133(35,4)$ & $118(36,1)$ & $251(35,7)$ \\
\hline Sec. común & $146(38,8)$ & $120(36,7)$ & $266(37,8)$ \\
\hline Sec. técnica & $3(0,8)$ & 0 & $3(0,4)$ \\
\hline Superior no & & & \\
\hline universitaria & $22(5,0)$ & $33(10,1)$ & $55(7.8)$ \\
\hline Universitaria & $21(5,6)$ & $24(7,3)$ & $45(6,4)$ \\
\hline Otro & 0 & $1(0,3)$ & $1(0,1)$ \\
\hline \multicolumn{4}{|l|}{ Estado civil: } \\
\hline Conviviente & $116(30,9)$ & $61(18,7)$ & $177(25,2)$ \\
\hline Casado(a) & $128(34,0)$ & $135(41,3)$ & $263(37,4)$ \\
\hline Viudo(a) & $31(8,2)$ & $33(10,1)$ & $64(9,1)$ \\
\hline Divorciado(a) & 0 & $2(0,6)$ & $2(0,3)$ \\
\hline Separado(a) & $15(4,0)$ & $27(8,3)$ & $42(6,0)$ \\
\hline Soltero & $86(22,9)$ & $69(21,1)$ & $155(22,0)$ \\
\hline \multicolumn{4}{|l|}{ Lengua materna: } \\
\hline Español & $255(67,8)$ & $263(80,4)$ & $518(73,7)$ \\
\hline Nativa & $121(32,2)$ & $64(19,6)$ & $185(26,3)$ \\
\hline Total & $376(100)$ & $327(100)$ & $703(100)$ \\
\hline
\end{tabular}

Sec.: Secundaria. 
El nivel educacional muestra tanto en CS como en hospitales que la mayor proporción de usuarios tenía nivel educacional secundario y primario (alrededor de un tercio), existiendo una ligera mayor proporción de población analfabeta $(11,7 \%)$ en los CS en relación a hospitales $(8,3 \%)$. La población de usuarios con nivel superior fue mayor en hospitales $(17,4 \%)$ que en los centros de salud (10,6\%). Da la impresión que la población de los hospitales tenía mayor nivel educacional.

Analizando el estado civil de los usuarios, se observó que $64,9 \%$ de usuarios de CS es casado o conviviente; en hospitales, esta población constituye el $60 \%$. Aproximadamente 1 de cada 5 usuarios es soltero, tanto en CS como en hospitales.

Una variable de interés fue la lengua materna del usuario; en el caso de los CS se encontró que 2 de cada 3 eran de lengua materna española, mientras que en hospitales, $80,4 \%$ era de aquella condición. La población lengua materna nativa fue mayor en CS $(32,2 \%)$ con relación a los hospitales $(19,6 \%)$.

Distribuyendo la población de análisis según nivel socioeconómico, se observó que $46 \%$ de los que acudieron a los CS no fueron pobres y $13,6 \%$ era pobre extremo, a diferencia de los que acudieron a los hospitalesm donde tales proporciones fueron $62,4 \%$ y $5,2 \%$, respectivamente (Tabla 2). Las poblaciones de CS y hospitales según NSE mostraron diferencias significativas (prueba U-Mann Whitney, $p<0,001)$.

Al determinar la accesibilidad geográfica, la distancia del hogar de los usuarios a los establecimientos de salud MINSA fue medida en función del tiempo que se requiere para llegar a ellos. Así, en el caso de los CS, $66,2 \%$ de los usuarios demoró menos de 15 minutos, mientras que 12,5\% necesitó más de 30 minutos para llegar. En el caso de los hospitales, 48,6\% empleó menos de 15 minutos y 24,8\% demoró más de 30 minutos (Tabla 2). La media del grupo CS fue 20,5 minutos (error estándar $=1,39$ ) y del grupo de hospitales fue 33,2 minutos (error estándar $=2,34)$. Estas poblaciones de CS y hospitales según distancia al establecimiento mostraron diferencias significativas (prueba U-Mann Whitney, $p<0,001$ ).

En la accesibilidad para la atención, se midió el tiempo de espera, vale decir el tiempo en minutos que el usuario una vez llegado al establecimiento tuvo que aguardar para ser atendido. La Tabla 2 muestra que, en los CS, $45,5 \%$ de usuarios esperó menos de 15 minutos para ser atendido y 13,8\% aguardó mas de una hora, a diferencia de los hospitales donde $31,8 \%$ esperó menos de 15 minutos y $28,7 \%$ más de una hora para la atención. La media para el grupo de CS fue 39,1 minutos (error estándar $=2,59$ ) y para los atendidos en el hospital 64,9 minutos (error estándar $=3,91$ ). Estas poblaciones de CS y hospitales según tiempo de espera para la atención mostraron diferencias significativas (prueba U-Mann Whitney, $p<0,001)$.

El $68,1 \%$ de los entrevistados de centros de salud manifestó que encontró satisfactoria la atención recibida, mientras que 105 usuarios $(27,9 \%)$ expresaron su poca satisfacción y un $4 \%$ su insatisfacción. A nivel de hospitales, $62,1 \%$ reportó satisfacción, la poca satisfacción llegó al 29,1\% y la insatisfacción al 8,9\%. La categoría de insatisfacción mostró diferencias significativas según tipo de establecimientos (Tabla 2).

Tabla 2. Distribución de variables sociodemográficas, de accesibilidad y de satisfacción de los usuarios según establecimientos de salud del MINSA (Perú, ENNIV 2000).

\begin{tabular}{ccc}
\hline Centros & & \\
de salud & Hospitales & Total \\
$\mathrm{n}(\%)$ & $\mathrm{n}(\%)$ & $\mathrm{n}(\%)$ \\
\hline
\end{tabular}

Nivel socio-económico

$\begin{array}{lccc}\text { No pobres } & 173(46,0) & 204(62,4) & 377(53,6) \\ \text { Pobres no extremos } & 152(40,4) & 106(32,4) & 258(36,7) \\ \text { Pobres extremos } & 51(13,6) & 17(5,2) & 68(9,7) \\ \text { Total } & 376(100) & 327(100) & 703(100)\end{array}$

Distancia desde el hogar al establecimiento de salud

$\begin{array}{lccc}<15 \text { minutos } & 249(66,2) & 159(48,6) & 408(58,0) \\ 15-30 & 80(21,3) & 87(26,6) & 167(23,8) \\ 30-60 & 30(8,0) & 50(15,3) & 80(11,4) \\ >60 \text { minutos } & 17(4,5) & 31(9,5) & 48(6,8) \\ \text { Total } & 376(100) & 327(100) & 703(100)\end{array}$

Tiempo de espera para la atención

$<15$ minutos $\quad 171(45,5) \quad 104(31,8) \quad 275(39,1)$

$15-30 \quad 86(22,9) \quad 63(19,3) \quad 149(21,2)$

30-60 $67(17,8) \quad 66(20,2) \quad 133(18,9)$

> 60 minutos $\quad 52(13,8) \quad 94(28,7) \quad 146(20,8)$

Total $376(100) \quad 327(100) \quad 703(100)$

Opinión del usuario sobre la atención

$\begin{array}{lccc}\text { Satisfactoria } & 256(68,1) & 203(62,1) & 459(65,3) \\ \text { Poco satisfactoria } & 105(27,9) & 95(29,1) & 200(28,4) \\ \text { No le satisfizo } & 15(4,0) & 29(8,9) & 44(6,3) \\ \text { Total } & 376(100) & 327(100) & 703(100)\end{array}$


Tabla 3. Porcentual de satisfacción con la atención en CS y hospitales MINSA según nivel económico de los usuarios (quintil de gastos per cápita anual). ENNIV 2000.

\begin{tabular}{llccc}
\hline Nivel económico & \multicolumn{1}{c}{$\begin{array}{c}\text { Categoría } \\
\text { SU }\end{array}$} & $\begin{array}{c}\text { Centros de salud } \\
\mathrm{n}(\%)\end{array}$ & $\begin{array}{c}\text { Hospitales } \\
\mathrm{n}(\%)\end{array}$ & $\begin{array}{c}\text { Total } \\
\mathrm{n}(\%)\end{array}$ \\
\hline \multirow{2}{*}{ No pobres } & Satisfactoria & $115(66,5)$ & $123(60,3)$ & $238(63,1)$ \\
& Poco satisfactoria & $53(30,6)$ & $61(29,9)$ & $114(30,2)$ \\
& No le satisfizo & $5(2,9)$ & $20(9,8)$ & $25(6,6)$ \\
& Total & $173(100)$ & $204(100)$ & $377(100)$ \\
& Satisfactoria & $103(67,8)$ & $64(60,4)$ & $167(64,7)$ \\
& Poco satisfactoria & $41(27,0)$ & $34(32,1)$ & $75(29,1)$ \\
& No le satisfizo & $8(5,3)$ & $8(7,5)$ & $16(6,2)$ \\
Pobres extremos extremos & Total & $152(100)$ & $106(100)$ & $258(100)$ \\
& Satisfactoria & $38(74,5)$ & $16(94,1)$ & $54(79,4)$ \\
& Poco satisfactoria & $11(21,6)$ & 0 & $11(16,2)$ \\
& No le satisfizo & $2(3,9)$ & $1(5,9)$ & $3(4,4)$ \\
& Total & $51(100)$ & $17(100)$ & $68(100)$ \\
\hline
\end{tabular}

En la Tabla 3 se muestra los resultados del grado de satisfacción de los usuarios según NSE. En el caso de los centros de salud, la mayor satisfacción, 74,5\%, ocurrió en los pobres extremos, en menor nivel en los pobres no extremos $(67,8 \%)$ y ligeramente más bajo aún en los no pobres $(66,5 \%)$. Para la categoría atención “poco satisfactoria", se observa una tendencia inversa, es decir, los mayores niveles se encuentran en los no pobres $(30,6 \%)$ y los más bajos en los pobres extremos $(21,6 \%)$.

En la población que acudió a los hospitales, se encontró que la satisfacción fue alrededor de $60 \%$, tanto a nivel de los no pobres como pobres no extremos. Adicionalmente, los pobres extremos mostraron $94,1 \%$ de satisfacción. La insatisfacción con la atención muestra una relación directa con el NSE. Así, en los no pobres es de 9,8\%, llegando a $7,5 \%$ en los pobres y $5,9 \%$ en los pobres extremos.

Para la categoría "atención satisfactoria”, el análisis chi cuadrado de tendencia entre el NSE según los establecimientos no muestra asociación estadística (chi cuadrado $=2,241$, valor $p=0,134$ ). Se comparó la relación entre las categorías pobres extremos y no pobres según CS y hospitales, encontrándose que los pobres extremos tienen más posibilidades de ser satisfechos en comparación con los pobres (chi cuadrado $=8,55, p=$ 0,034, OR mantel hanzstel 2,54, IC 95\% 1,28-5,05)

La Tabla 4 muestra los resultados del análisis multivariado para centros de salud, es decir, se presenta la relación ajustada entre la satisfacción de usuarios y las variables independientes. El NSE muestra cierta tendencia a relacionarse con el grado de satisfacción, es decir, los más pobres tendrían mayor satisfacción; sin embargo, tal impresión no es estadísticamente significativa.

En el caso del nivel educacional, los de nivel superior tienen menor porcentaje de satisfacción con relación a los que no tienen estudios, según resultados del análisis de frecuencia simple. Sin embargo, esa relación no fue significativa al ajustar efectos $(\mathrm{OR}=0,54$, IC $95 \% 0,19$ 1,58 , valor $p=0,265$ ). Empero existe cierta tendencia asociativa. Inversamente, los de menor nivel educacional presentaron más posibilidades de satisfacción, aunque en magnitudes no significativas.

El factor edad muestra asociación positiva con la satisfacción, es decir, a mayor edad existe más probabilidad de estar satisfecho con la atención. Por ejemplo, los mayores de 60 años tuvieron 4 veces más probabilidades de estar satisfechos en relación a los adolescentes $(\mathrm{OR}=$ $4,03$, IC 95\% 1,48-11,01, valor $p=0,006)$. De otro lado, los no unidos (solteros, divorciados) presentaron mayor probabilidad de ser satisfechos con la atención (OR $=2,27$, IC 95\% 1,25-4,12, valor $p=0,007)$. No se encontró asociaciones entre la lengua materna y el sexo del usuario con la satisfacción.

La distancia al establecimiento muestra cierta asociación inversa con el nivel de satisfacción, es decir, menores distancias se reflejan en mayor SU según los resultados del análisis de frecuencia simple, sobretodo para las categorías extremas (distancia menor de 15 
Tabla 4. Factores asociados con la satisfacción de usuarios en centros de salud MINSA (n = 376). ENNIV 2000.

\begin{tabular}{|c|c|c|c|c|c|}
\hline Factor de estudio & $\begin{array}{c}\text { Frecuencia } \\
(\%)\end{array}$ & $\begin{array}{l}\text { Razòn de posibilidades } \\
\text { cruda (IC 95\%) }\end{array}$ & $\begin{array}{c}\text { Valor } \\
p\end{array}$ & $\begin{array}{l}\text { Razòn de posibilidades } \\
\text { ajustada (IC 95\%) }\end{array}$ & $\begin{array}{c}\text { Valor } \\
p\end{array}$ \\
\hline \multicolumn{6}{|l|}{ Nivel económico } \\
\hline No pobres & 46,0 & 1,00 & & 1,00 & \\
\hline Pobres extremos & 13,6 & $1,47(0,73-2,98)$ & 0,280 & $1,30(0,59-2,85)$ & 0,510 \\
\hline Pobres no extremos & 40,4 & $1,06(0,67-1,69)$ & 0,805 & $1,04(0,63-1,74)$ & 0,868 \\
\hline \multicolumn{6}{|l|}{ Nivel educacional } \\
\hline Ninguno & 11,7 & 1,00 & & 1,00 & \\
\hline Primaria & 37,2 & $1,59(0,77-3,27)$ & 0,206 & $1,91(0,83-4,40)$ & 0,130 \\
\hline Secundaria & 39,7 & $1,28(0,63-2,59)$ & 0,494 & $1,50(0,61-3,69)$ & 0,378 \\
\hline Superior & 11,4 & $0,60(0,25-1,41)$ & 0,241 & $0,54(0,19-1,58)$ & 0,265 \\
\hline \multicolumn{6}{|l|}{ Grupos etáreos } \\
\hline 16-19 años & 11,9 & 1,00 & & 1,00 & \\
\hline $20-39$ & 39,1 & $1,77(0,89-3,52)$ & 0,106 & $3,37(1,45-7,83)$ & 0,005 \\
\hline $40-59$ & 30,9 & $1,29(0,64-2,60)$ & 0,481 & $2,68(1,08-6,63)$ & 0,033 \\
\hline$>60$ años & 18,1 & $2,37(1,05-5,36)$ & 0,037 & $4,03(1,48-11,01)$ & 0,006 \\
\hline \multicolumn{6}{|l|}{ Estado civil } \\
\hline Unido & 64,9 & 1,00 & & 1,00 & \\
\hline No unido & 35,1 & $1,48(0,93-2,36)$ & 0,099 & $2,27(1,25-4,12)$ & 0,007 \\
\hline \multicolumn{6}{|l|}{ Lengua materna } \\
\hline Nativo & 32,2 & 1,00 & & 1,00 & \\
\hline Español & 67,8 & $1,21(0,76-1,91)$ & 0,423 & $1,26(0,75-2,13)$ & 0,378 \\
\hline \multicolumn{6}{|l|}{ Sexo } \\
\hline Mujer & 57,7 & 1,00 & & 1,00 & \\
\hline Hombre & 42,3 & $0,94(0,61-1,46)$ & 0,779 & $0,89(0,55-1,45)$ & 0,637 \\
\hline \multicolumn{6}{|c|}{ Distancia al establecimiento } \\
\hline$>60$ minutos & 4,5 & 1,00 & & 1,00 & \\
\hline$<15$ & 66,2 & $1,95(0,73-5,24)$ & 0,186 & $1,76(0,60-5,20)$ & 0,305 \\
\hline $15-30$ & 21,3 & $1,75(0,61-5,00)$ & 0,303 & $1,73(0,55-5,50)$ & 0,352 \\
\hline 30-60 minutos & 8,0 & $2,92(0,82-10,44)$ & 0,990 & $2,79(0,71-11,01)$ & 0,144 \\
\hline \multicolumn{6}{|l|}{ Tiempo de espera } \\
\hline$>60$ minutos & 13,8 & 1,00 & & 1,00 & \\
\hline$<15$ & 45,5 & $2,72(1,42-5,20)$ & 0,003 & $2,86(1,43-5,71)$ & 0,003 \\
\hline $15-30$ & 22,9 & $1,60(0,79-3,23)$ & 0,190 & $1,75(0,83-3,70)$ & 0,140 \\
\hline 30-60 minutos & 17,8 & $1,44(0,69-3,01)$ & 0,332 & $1,54(0,70-3,39)$ & 0,278 \\
\hline
\end{tabular}

minutos versus mayores de 60 minutos). Aún así, el análisis multivariado revela que dicha tendencia no es significativa.

En el análisis de frecuencia simple, se encontró asociación entre el tiempo de espera para la atención y SU; según los resultados del mutivariado la población que esperó menos de 15 minutos para ser atendida tuvo 2 veces más probabilidades de estar satisfecha en relación con la que esperó más de 60 minutos $(\mathrm{OR}=2,86$, IC $95 \%=1,43-5,71$, valor $p=0,003)$. Para el resto de categorías, no se encontró asociación.

Los resultados del análisis multivariado para la población de hospitales se muestra en la Tabla 5. Para el caso del NSE, se observa que los pobres extremos tienen más probabilidades de estar satisfechos en relación a los no pobres $(\mathrm{OR}=11,5$, IC $95 \%=1,44-92,35$, valor $p$ $=0,021)$.

El nivel educacional muestra cierta asociación positiva con la SU. Empero, la significancia estadística es valedera para el caso de la población de estudios superiores, es decir, estos últimos tienen aproximadamente 3 veces mayores posibilidades de estar satisfechos con relación a los que no tienen estudios (OR $=2,93$, IC $95 \%=$ $1,35-8,40$, valor $p=0,041$ ).

Al analizar la relación de los grupos etáreos con la SU, la ligera tendencia de asociación encontrada en el 
Tabla 5. Factores asociados con la satisfacción de usuarios en hospitales MINSA (n =327). ENNIV 2000.

\begin{tabular}{|c|c|c|c|c|c|}
\hline Factor de estudio & $\begin{array}{c}\text { Frecuencia } \\
(\%)\end{array}$ & $\begin{array}{l}\text { Razòn de posibilidades } \\
\text { cruda (IC 95\%) }\end{array}$ & $\begin{array}{l}\text { Valor } \\
p\end{array}$ & $\begin{array}{l}\text { Razòn de posibilidades } \\
\text { ajustada (IC 95\%) }\end{array}$ & $\begin{array}{l}\text { Valor } \\
\quad p\end{array}$ \\
\hline \multicolumn{6}{|l|}{ Nivel económico } \\
\hline No pobres & 62,4 & 1,00 & & 1,00 & \\
\hline Pobres extremos & 5,2 & $10,53(1,37-80,9)$ & 0,024 & $11,52(1,44-92,35)$ & 0,021 \\
\hline Pobres no extremos & 32,4 & $1,01(0,62-1,62)$ & 0,989 & $1,01(0,60-1,66)$ & 0,957 \\
\hline \multicolumn{6}{|l|}{ Nivel educacional } \\
\hline Ninguno & 8,3 & 1,00 & & 1,00 & \\
\hline Primaria & 37,3 & $1,72(0,74-3,97)$ & 0,206 & $1,95(0,79-4,84)$ & 0,147 \\
\hline Secundaria & 36,7 & $1,86(0,80-4,3)$ & 0,148 & $2,19(0,82-5,86)$ & 0,120 \\
\hline Superior & 17,7 & $2,21(0,87-5,6)$ & 0,096 & $2,93(1,35-8,40)$ & 0,041 \\
\hline \multicolumn{6}{|l|}{ Grupos etáreos } \\
\hline 16-19 años & 8,6 & 1,00 & & 1,00 & \\
\hline $20-39$ & 35,8 & $0,80(0,34-1,89)$ & 0,607 & $1,02(0,39-2,63)$ & 0,973 \\
\hline $40-59$ & 31,5 & $1,13(0,47-2,7)$ & 0,788 & $1,79(0,66-4,90)$ & 0,253 \\
\hline > 60 años & 24,2 & $0,82(0,33-1,99)$ & 0,656 & $1,60(0,56-4,52)$ & 0,379 \\
\hline \multicolumn{6}{|l|}{ Estado civil } \\
\hline Unido & 59,9 & 1,00 & & 1,00 & \\
\hline No unido & 40,1 & $1,29(0,82-2,04)$ & 0,277 & $1,42(0,83-2,42)$ & 0,198 \\
\hline \multicolumn{6}{|l|}{ Lengua materna } \\
\hline Nativo & 19,6 & 1,00 & & 1,00 & \\
\hline Español & 78,9 & $1,59(0,91-2,8)$ & 0,101 & $1,52(0,82-2,83)$ & 0,182 \\
\hline \multicolumn{6}{|l|}{ Sexo } \\
\hline Mujer & 58,7 & 1,00 & & 1,00 & \\
\hline Hombre & 41,3 & $1,19(0,75-1,88)$ & 0,460 & $1,14(0,70-1,87)$ & 0,590 \\
\hline \multicolumn{6}{|c|}{ Distancia al establecimiento } \\
\hline$>60$ minutos & 9,5 & 1,00 & & 1,00 & \\
\hline$<15$ & 48,6 & $0,44(0,18-1,09)$ & 0,077 & $0,37(0,14-0,97)$ & 0,044 \\
\hline $15-30$ & 26,6 & $0,39(0,15-1,01)$ & 0,053 & 0,39 & 0,066 \\
\hline 30-60 minutos & 15,3 & $0,60(0,20-1,58)$ & 0,277 & 0,57 & 0,307 \\
\hline \multicolumn{6}{|l|}{ Tiempo de espera } \\
\hline$>60$ minutos & 28,7 & 1,00 & & 1,00 & \\
\hline$<15$ & 31,8 & $1,03(0,58-1,84)$ & 0,908 & $1,02(0,54-1,89)$ & 0,962 \\
\hline $15-30$ & 19,3 & $1,16(0,59-2,25)$ & 0,668 & $1,08(0,54-2,18)$ & 0,820 \\
\hline 30-60 minutos & 20,2 & $0,90(0,47-1,71)$ & 0,739 & $0,89(0,44-1,78)$ & 0,737 \\
\hline
\end{tabular}

análisis de frecuencia simple no muestra significancia alguna con el ajuste estadístico.

No se encontró asociaciones significativas entre el estado civil, lengua materna y el sexo con la satisfacción. Aun cuando en el análisis de frecuencia simple se observó que la población lengua materna nativa atendida en hospitales expresó menor nivel de satisfacción.

Con relación a la distancia al establecimiento, se encuentra que aquellos que viven a menos de 15 minutos del hospital tienen $59 \%$ menos probabilidades de estar satisfechos en comparación con los que viven a una distancia mayor de 60 minutos $(\mathrm{OR}=0,37$, IC 95\% $=$ $0,14-0,97$, valor $p=0,044)$, tendencia similar aunque no significativa se observa para los que viven a 15 ó 30 minutos.

El tiempo de espera para la atención no mostró relación alguna con la satisfacción del usuario.

\section{DISCUSIÓN}

La información obtenida en el presente análisis corresponde a datos recolectados en el año 2000, momento histórico en que el Perú atravesaba una serie de conflictos político sociales que pretendían restaurar el orden democrático. Dicho periodo marcó un punto de quiebre político institucional y de transición gubernamental relevante, que tendría que ser 
considerado como referencia durante la lectura de nuestros hallazgos.

Es importante resaltar el hecho de que las poblaciones que acudieron a los establecimientos MINSA son diferentes en sus características sociodemográficas y de accesibilidad. Así, los que acudieron a los CS fueron, en general y comparados con la población hospitalaria, de menor edad, de menor nivel educacional, en mayor proporción hijos de madres que hablan lengua diferente al español y de menor nivel económico. Adicionalmente, están más próximos de los CS y esperan menos tiempo para atenderse. Esto sustenta la idea de diferenciales según lugar de atención para el estudio e interpretación de la SU.

La satisfacción del usuario es un tema de interés creciente en la salud pública y gestión de servicios $\left({ }^{23}\right)$. Su conceptualización y entendimiento causal es complejo y múltiple, primero, porque reside en la percepción subjetiva del usuario y, luego, porque se basa en metodologías, técnicas e instrumentos de medición diversos $\left({ }^{24,25,26,27,28}\right)$ y poco estandarizados $\left.{ }^{(29}\right)$. De otro lado, es cada vez mayor la presión social por servicios de salud accesibles y de calidad que atiendan las necesidades de la población. Desde hace algunos años, el estudio de la SU y de sus expectativas es considerado uno de los métodos de evaluación de los servicios de salud $\left({ }^{30}\right)$ e interpretado como resultado de la calidad de la asistencia $\left({ }^{31}\right)$ y de su efectividad $\left({ }^{21}\right)$. Adicionalmente, se ha encontrado relación entre la satisfacción, la utilización de los servicios ${ }^{32}$ ), la conducta terapéutica del paciente, su continuidad y resultados en salud $\left({ }^{33}\right)$.

El presente estudio encontró que la satisfacción de usuarios fue de $68 \%$ en centros de salud, siendo un poco menor en hospitales. El grado de SU de los CS es un poco menor que lo reportado por Hall y Dornan, quienes a través de un metaanálisis reportaron que el nivel promedio de satisfacción en poco más de 200 estudios, en su mayoría descriptivos, realizados en consultorios clínicos ambulatorios fue de $76 \%\left({ }^{34}\right)$. En otro estudio cuantitativo transversal realizado en servicios obstétricos de 37 hospitales del Perú utilizando la escala de Likert, se encontró niveles de satisfacción entre 55 y $67 \%$ ( $\left.{ }^{35}\right)$. Otro estudio puntual en un hospital peruano encontró altos niveles de satisfacción de expectativas de gestantes que acudieron a control prenatal ${ }^{\left({ }^{36}\right)}$. Estas diferencias podrían obedecer a metodologías de estudio también diferentes. Sin embargo, es oportuno señalar que existe la tendencia a medir satisfacción no sólo desde el punto de vista global sino, mas bien, analizarla durante todo el proceso de atención en salud $\left(^{6}\right)$, lo cual facilitaría la identificación de aspectos útiles en términos de desarrollar esfuerzos colectivos hacia la mejora de la atención. La encuesta ENNIV, debido a que trata de investigar los resultados de la implementación de políticas sanitarias del Perú para un periodo determinado (satisfacción con "enfoque resultado"), no exploró aspectos específicos del proceso de atención. Una vez más se ejemplifica que el objetivo del investigador / gestor / político/ decisor en salud es quien define la metodología de la investigación, tal como fue señalado por Habicht y colaboradores $\left({ }^{37}\right)$. La escala de medición utilizada en el presente estudio fue ordinal con 3 categorías, lo cual representa sólo uno de los métodos internacionalmente usados en SU. Antes bien, existe una tendencia reciente hacia la utilización de escalas basadas en puntuaciones numéricas (scores) que evalúan el desempeño de los servicios de salud según la opinión de los usuarios. Estas escalas permiten identificar áreas susceptibles de mejoría ${ }^{\left({ }^{8}\right)}$. Adicionalmente, otro método para medir satisfacción es aquel que se basa en el balance de las percepciones del usuario durante su visita al establecimiento en función de sus expectativas, el instrumento SERVQUAL se basa en dicho enfoque. El SERVQUAL se dirige hacia una mejoría organizacional del servicio (SU como "enfoque proceso"), tomando como referencia las brechas percepción / expectativas para un conjunto de aspectos de la atención (tangibilidad, capacidad de respuesta, confiabilidad, seguridad, empatía) $\left.{ }^{39}\right)$. Dicho enfoque fue aplicado por investigadores en algunos centros de salud del Perú ("estudios de caso") $\left({ }^{40,41,42,43,44}\right)$ y hospitales $\left({ }^{45}\right)$, encontrándose altos niveles de insatisfacción, lo que podría ser interpretado como contradictorio con nuestros hallazgos. Sin embargo, el método de nuestro estudio aborda la SU como "enfoque resultado", no explorando en lo absoluto las expectativas del usuario, lo cual minimiza sustantivamente las posibilidades de comparación.

Se exploró la relación entre la satisfacción y el NSE. Desde la óptica de reducción de desigualdades en salud, se ha desarrollado esfuerzos por medir la satisfacción en poblaciones de bajos recursos y con limitado acceso a servicios de salud públicos, en la idea de identificar brechas de disparidad $\left({ }^{46}\right)$, las cuales son de referencia para la formulación y aplicación de políticas sanitarias $\left({ }^{47}\right)$. Se ha encontrado que las poblaciones de menores ingresos tienen menores niveles de satisfacción, sobre todo cuando ellas asisten a hospitales públicos. Inversamente, las poblaciones 
de mayores ingresos tienden a presentar menor satisfacción cuando son atendidas en CS. Ese aspecto ha sido asociado con los mayores niveles de expectativas que tendrían las personas de mayores ingresos económicos y su poca satisfacción con la atención de un establecimiento no "especializado" como el centro de salud. En el presente estudio existe cierta tendencia a favor de esa relación, es decir, en los CS los no pobres tienden a menor satisfacción, aunque en niveles no significativos. En el caso de hospitales e incluso en centros de salud, los pobres extremos tienen más probabilidades de satisfacción. Contrariamente, Hall y Dornan encontraron, en población de habla inglesa, que los usuarios de mayores ingresos muestran mayores niveles de satisfacción $\left({ }^{48}\right)$. La diferencia podría obedecer al método de valoración de la pobreza. Nuestro estudio utilizó la línea de pobreza que se basa en el nivel de gastos per cápita, en tanto Hall y Dornan lo hicieron utilizando el nivel de ingresos. Adicionalmente, es probable que existan complejas interacciones entre NSE y otros factores no abordados por este estudio, probablemente de índole sociocultural, que explicarían esta discrepancia.

La población con nivel educacional superior tuvo menores niveles de satisfacción cuando fue atendida en centros de salud. Lo opuesto ocurrió en el caso de hospitales, donde la relación nivel educacional y satisfacción fue directa, es decir, los de nivel superior presentaron mayor satisfacción. Este aspecto reflejaría la mayor afinidad de los letrados por servicios de mayor complejidad y con acceso a especialidades médicas, procedimientos de avanzada y exámenes auxiliares que se sitúan principalmente en hospitales. Es probable que esta población tenga expectativas de mayor complejidad tecnológica y las vea satisfechas durante su periplo hospitalario.

El caso de la población de menor educación atendida en CS podría ser interpretado de modo distinto. Para este grupo, el acceso y uso de servicios de salud tiene un rol importante en la satisfacción. Ese solo hecho ya lo satisface en gran medida. Al parecer, la concepción del proceso de atención de su problema de salud no considera complejidad tecnológica, sino mas bien el "ser atendido", tratado, cordialmente y en sintonía con sus pareceres y creencias; y que, lógicamente, sus problemas de salud ("generalmente menos severos") sean resueltos. Empero, los niveles de satisfacción en hospitales para este grupo es menor, debido, entre otros factores, al distanciamiento sociocultural, la provisión de servicios que escapan a su cosmovisión y racionalidad local y la poca calidez recibida del personal de salud. Es muy probable que las poblaciones de menor educación se sientan más "afines" social, cultural y antropológicamente con el centro de salud (sobre todo con su personal, ya que en muchos casos ellos son de su mismo entorno), lo que resultaría en relaciones de mayor confianza y comunicación que contribuirían a una mayor satisfacción. Sin embargo, estos aspectos merecerían ser estudiados más profundamente y tomados en cuenta en el momento de la planificación y organización de los servicios de salud.

Hall y Dornan encontraron en su metaanálisis que la edad es un importante factor asociado a SU, afirmando que las poblaciones más longevas tienden a mayor SU. Similar relación fue encontrada en un estudio realizado en Suecia $\left({ }^{49}\right)$. Incluso en un estudio longitudinal, la edad mantenía su asociación con la SU durante las visitas de seguimiento $\left({ }^{17}\right)$. En el presente estudio, la relación de la SU con la edad para el caso de centros de salud y hospitales es lineal; mayor satisfacción se observó en usuarios de más edad. Ello tendría relación con la propia concepción sobre el proceso salud-enfermedad de las poblaciones jóvenes: percepción de falta de vulnerabilidad para enfermar, cuestionamiento a algunas de las medidas diagnósticas y terapéuticas, exigencia de resolución inmediata y altas expectativas. Todo ello se reflejaría en una menor satisfacción. Esa concepción va cambiando en el transcurso del ciclo biológico. Así, las poblaciones de mayor edad son concientes de su vulnerabilidad para enfermar y ven que la atención es un proceso de interacción y responsabilidad compartida con el servicio, y donde su participación es crucial para resolver sus problemas. Adicionalmente, el actual perfil demográfico y epidemiológico de la población en el mundo nos muestra que existe una mayor prevalencia de enfermedades crónicas y degenerativas a medida que la edad avanza $\left({ }^{50}\right)$. Ellas requieren para su cuidado integral un contacto regular con los servicios de salud. Esa mayor continuidad en la atención permitiría que a la larga se establezca una relación de confianza, mayor afinidad y seguridad, importante entre el usuario y el servicio, lo cual podría evidenciarse en una mayor satisfacción. Esta situación no ocurre con las poblaciones más jóvenes, las cuales adolecen fundamentalmente de problemas agudos o de rápida resolución. El acceso a medicamentos también ha sido señalado como asociado positivamente a la satisfacción ${ }^{(21)}$; el manejo de los problemas de salud de las personas de mayor edad requiere mayor prescripción de medicamentos, lo cual contribuiría a una mayor satisfacción. 
Otro factor asociado a satisfacción lo constituye la etnicidad / raza, existiendo datos que revelan que poblaciones de etnias nativas tienen menores niveles de satisfacción y reciben discriminación $\left({ }^{47}\right)$, más aún en hospitales. En nuestro estudio, usamos una variable próxima a etnicidad / raza, denominada lengua materna no española ("nativa"). No se encontró asociación significativa de esta variable con la satisfacción, sólo se apreció cierta tendencia hacia la menor SU en hospitales. Es probable que nuestra medición requiera de mayor validez y fineza. Aún cuando existe asociación de la variable LM nativa con menor NSE y de esta última con la satisfacción, no se encontró relación entre SU y LM nativa, lo que puede interpretarse como que existe una interacción compleja, donde intervienen probablemente otras variables plausibles de ser estudiadas en más detalle, sobretodo con el apoyo de técnicas cualitativas. Sería de utilidad profundizar este aspecto, ya que disparidades en el proceso de atención influyen en disparidades de resultados en salud, lo cual iría en sentido inverso con la macro política internacional ("metas del milenio") de reducción de las desigualdades y disparidades en el acceso y atención de salud. Con todo, se precisa de adaptaciones organizacionales en los establecimientos de salud públicos que garanticen no sólo el acceso a la atención sino, mas bien, procesos de calidad basados en la cordialidad, empatía, adaptación cultural y resolución compartida de problemas, en la perspectiva de contribuir hacia mejores niveles de salud de esta población particular.

Existen algunos reportes que enfatizan la mayor satisfacción de las mujeres con relación a los hombres. En el presente estudio tal asociación no fue encontrada. Probablemente, dentro de la red causal de la SU, para el caso peruano, existen otros factores de mayor relevancia.

El factor distancia al establecimiento de salud y su relación con la SU ha sido motivo de discusiones de interés; existe evidencia que la distancia influye en el uso de servicios $\left(^{51}\right)$. Para el caso de la satisfacción, se postula que los establecimientos menos accesibles en términos geográficos se asocian a menores niveles de satisfacción. El presente estudio encontró que la población que se atendió en CS tuvo una tendencia, estadísticamente no significativa, de asociación negativa entre distancia al establecimiento y SU (es decir, menores distancias se reflejan en mayores niveles de satisfacción). En el caso de hospitales, tal asociación es positiva (mayores distancias se traducen en mayor satisfacción), lo cual va en contraposición a lo publicado. Esto último podría obedecer a que el número de entrevistados por categoría de estudio fue insuficiente; que aquellos que vienen de más lejos hayan decidido atenderse en el hospital por prestigio institucional, recomendaciones de personas cercanas, entre otros, o que efectivamente los más alejados del hospital por el sólo hecho de tener acceso a la atención ya tienen una predisposición a la satisfacción. Asimismo, es factible que variables adicionales de tipo intermediarias o facilitadoras aún no exploradas estén actuando. Sin embargo, se requiere de ulteriores estudios para esclarecer estas hipótesis.

Existe reportes que exploran la relación entre la SU y el tiempo de espera para la atención, encontrándose una asociación inversa, es decir, menores tiempo de espera hacen esperar mayores niveles de satisfacción $\left({ }^{9}\right)$. Nuestros resultados son coincidentes con ello, especialmente para centros de salud. Es probable que las condiciones de angustia e incertidumbre que llevan consigo los pacientes que acuden a los establecimientos sean parcialmente superadas con la pronta atención y se traduzcan en una mayor probabilidad de satisfacción. Para el caso de los hospitales, la situación es distinta; existiría una mayor "capacidad de tolerancia" de los usuarios hacia prolongados tiempos de espera en la esperanza de tener una atención de mayor tecnología y resolución. Esto último podría tener mayor valorización en términos de satisfacción. De hecho, la población que acude a hospitales valoriza fundamentalmente aspectos relacionados con la competencia técnica, comunicación e información y la satisfacción de sus expectativas como aspectos cruciales de la atención.

Como puede verse, el estudio de la satisfacción y su entendimiento causal es complejo, multifactorial y dinámico, se encuentra estrechamente relacionado a aspectos subjetivos y objetivos, lo que posibilita la sinergia de los métodos cuantitativos y cualitativos para su mejor abordaje y estudio. Las variables sociodemográficas, económicas y de accesibilidad para la atención explican tan sólo una parte del fenómeno.

El presente estudio se basa en el análisis secundario de una encuesta nacional representativa para estudiar las condiciones y niveles de vida de la población peruana. Por tanto, tiene las limitaciones inherentes a la representatividad sobre el objeto de estudio, satisfacción con la atención; recordemos que el estudio no fue diseñado específicamente para este propósito. Sin embargo, los alcances y resultados del presente análisis permiten tener una idea aproximada sobre los objetivos 
planteados y abren espacios de discusión, utilización de información e identificación de nuevas rutas de pesquisa. La ENNIV responde a una iniciativa global de los gobiernos y organismos internacionales interesados en políticas sociales por hacer seguimiento y evaluar los resultados y avances de las políticas de desarrollo social de un país. Su propósito es la evaluación de resultados; por tanto, sus indicadores y preguntas de pesquisa se orientan hacia ello. En el caso del rubro "salud", la ENNIV pretendió investigar el estado de salud de la población peruana, su acceso a servicios, el lugar de atención y su resolución ante los problemas sanitarios, modos de financiamiento, entre otros aspectos. Recientemente, se incluyó el estudio de la satisfacción de usuarios como "enfoque resultado".

Es evidente que la exploración de la satisfacción no puede restringirse a una única pregunta si es que la idea hubiese sido la de obtener información para la mejora organizacional de los servicios de salud. Ello habría requerido explorar la satisfacción ante un conjunto de aspectos relacionados con la atención (confortabilidad, trato, cordialidad, empatía, nivel de información, privacidad, resolución del problema, acceso a medicamentos, costos, entre otros). Similarmente, la escala de medición de la satisfacción fue una ordinal (tres categorías: nivel satisfactorio, poco satisfactorio y no satisfactorio), lo cual limita el estudio y un análisis más fino del objeto de estudio. Existen informes que, al medir la satisfacción en escalas cuantitativas $\left({ }^{29,52}\right)$ o en escala Likert de cinco categorías $\left({ }^{18,21,53}\right)$, permiten una mayor profundidad del análisis de predictores y factores asociados. Otra limitante del estudio fue no explorar las expectativas de los usuarios. Existen datos que sustentan la relevancia de las expectativas en la satisfacción $\left({ }^{17}\right)$. Asimismo, ya fue señalada la aparente limitada validez de la variable lengua materna como para explorar la relación entre etnia/ raza y SU. No se consiguió analizar el comportamiento de la SU y sus factores asociados según regiones geográficas, lo cual fue nuestro deseo inicial, debido sobretodo a la reducida muestra para las categorías de la variable satisfacción en cada una de las regiones.

La encuesta fue dirigida a determinar el nivel de satisfacción, mas no así las razones o motivos de satisfacción o no satisfacción; estos aspectos hubiesen sido de enorme importancia durante el proceso de interpretación de los hallazgos y planteamiento de alternativas de abordaje. Por todo ello, no es posible establecer con total certeza inferencia de los resultados.
Pero, estos resultados dan una luz de información sobre un tema poco explorado en Perú.

Entonces, existen factores sociodemográficos, económicos y de accesibilidad para la atención que muestran relación con la satisfacción del usuario. Tales factores se comportan de manera particular según el tipo de establecimiento de salud (centro de salud, hospital), debido fundamentalmente a que la población usuaria es distinta. Esto último relativiza la comparación del nivel de satisfacción y de sus factores asociados entre tales establecimientos.

Específicamente, podemos concluir que en los CS existe tendencia de la población de menor NSE a mostrar mayores niveles de SU; la edad muestra asociación positiva con la satisfacción, las personas no unidas muestran mayor $\mathrm{SU}$; los de menor nivel educacional tienden a mostrar mayor SU; existe una asociación negativa entre el tiempo de espera para la atención y la satisfacción; igual comportamiento se observó con la distancia al establecimiento de salud. En el caso de hospitales, la población de menor NSE muestra una mayor predisposición a la satisfacción; el nivel educacional y la edad mostraron asociación positiva con la SU. La población lengua materna nativa expresa menor satisfacción con la atención; menores distancias al establecimiento de salud se asociaron con mayores niveles de satisfacción; no se encontró relación de la SU con el tiempo de espera.

Estos resultados podrían resultar en toma de decisiones de los gestores en términos de:

- Considerar la SU como un indicador para medir los resultados de políticas en salud destinadas a promover y garantizar la calidad en los servicios de salud del MINSA, donde la SU constituya un eje clave. Dichas políticas deberían lograr un balance racional con el enfoque basado en la productividad, tan de moda en la década de los 90.

- Establecer un sistema de monitoreo de la satisfacción y sus causales, identificando las experiencias de los pacientes durante su itinerario en el establecimiento de salud (SU como "enfoque proceso"), promoviendo estrategias de uso de información entre los gestores y el personal de salud.

- Promover el uso periódico y racional de las bases de datos de encuestas nacionales de tipo social y sanitario, pues, como se nota en este estudio, su análisis secundario puede contribuir en el mayor conocimiento de problemas relacionados con la salud y los efectos 
de las políticas públicas. Es loable la decisión de las instituciones de gobierno que, en un afán democrático, divulgan libremente dicha información vía web.

- Desde el punto de vista metodológico y con la idea de soporte a la gestión local, sería necesario realizar encuestas rápidas de medición de las experiencias, expectativas y satisfacción de los usuarios. De suma utilidad sería combinar los métodos cuantitativos y cualitativos. Ello facilitaría no sólo determinar la magnitud de la situación problema (el ¿cuánto?), sino también identificar las razones de dicha situación (el ¿por qué?).

\section{AGRADECIMIENTOS}

Especial agradecimientos a Ernesto Gozzer, Diego González Del Carpio, Alejandro Llanos y Luis Lozano, docentes de la Facultad de Salud Pública de la Universidad Peruana Cayetano Heredia, por sus valiosos aportes y sugerencias al documento. Igualmente, a la Lic. Gaby Caro por su esfuerzo en la revisión bibliográfica.

\section{REFERENCIAS BIBLIOGRÁFICAS}

1. Schneider H, Palmer N. Getting to the truth? Researching user views of primary health care. Health Policy Plann. 2002;17(1):32-41.

2. Mira J, Aranaz J. La satisfacción del paciente como una medida de resultado de la atención sanitaria. Med Clin (Barc). 2000;114(supl 3):26-33.

3. Edwards $\mathrm{C}$, Staniszewska S. Accessing the user's perspective. Health Soc Care Community. 2000;8(6):417-24.

4. Traverso-Yèpez m, Morais NA. Reinvindicando a subjetividade dos usuarios da Rede Básica de Saùde: para uma humanizacao do atendimento. Cad. Saùde Pùblica. 2004;20(1):80-8.

5. Pascoe G. Patient satisfaction in primary care: a literature review and analysis. Evaluation and Program Planning. $1983 ; 6: 185-210$

6. Wensing M, Elwyn G. Research on patients' views in the evaluation and improvement of quality of care. Qual Saf Health Care. 2002;11:153-7.

7. Kane RL, Maciejewski M, Finch M. The relationship of patient satisfaction with care and clinical outcomes. Med Care. 1997;35:714-30.

8. Jenkinson C, Coulter A, Bruster S, Richards N, Chandola. Patients' experiences and satisfaction with health care: results of a questionnaire study of specific aspects of care. Qual Saf Health Care. 2002;11:335-9.

9. Fitzpatrick R. Surveys of patient satisfaction: important general considerations. Br Med J. 1991;302:887-9.

10. Ministerio de Salud del Perú, Proyecto 2000. Calidad del servicio de salud desde la percepción de la usuaria:
Diagnóstico de necesidades de comunicación. Lima: Pathfinder International, USAID; 2002.

11. Williams B, Coyle J, Healy D. The meaning of patient satisfaction: an explanation of high reported levels. Soc Sci Med. 1998;47(9):1351-9.

12. Jackson J, Chamberlin J, Kroenke K. Predictors of patient satisfaction. Soc Sci Med. 2001;52:609-20.

13. Baron-Epel O, Dushenat M, Friedman N. Evaluation of the consumer model: relationship between patients' expectations, perceptions and satisfaction with care. Int $\mathbf{J}$ Qual Health Care. 2001;13(4):317-23.

14. Sitzia J, Wood N. Patient satisfaction: a review of issues and concepts. Soc Sci Med. 1997;45(12):1829-43.

15. Mendoza J, Piechulek H, Al-Sabir A. Client satisfaction and quality of health care in rural Bangladesh. Bull WHO. 2001;79(6):512-7.

16. Baltussen R, Yè Y, Hadad S, Sauerborn R. Perceived quality of care of primary care services in Burkina Faso. Health Policy and Plann. 2002;17(1):42-8.

17. Gross R, Tabenkin H, Porta A, Heymann A, Greenstein M, Porter B, Matzliach R. The relationship between primary care physicians' adherence to guidelines for the treatment of diabetes and patient satisfaction: findings from a pilot study. Fam Pract. 2003;20(5):563-9.

18. Bernhart M, Wiadnyana I, Wihardjo H, Pohan I. Patient satisfaction in developing countries. Soc Sci Med. 1999;48:989-96.

19. Ministerio de Salud del Perú, Proyecto 2000. Proyecto 2000: Un proyecto de innovaciones en salud. Reporte técnico final del equipo de asistencia técnica. Lima: Ministerio de Salud del Perú, Proyecto 2000; 2002.

20. Ministerio de Salud del Perú. Lineamientos de políticas del sector salud 1995-2000. Lima: Ministerio de Salud del Perú; 2001 [Accesado 20 octubre 2001]. Disponible en: http:// www.minsa.gob.pe/publicaciones/pubs/lineamientos/ index.htm

21. Ministerio de Salud del Perú. Lineamientos de políticas sanitarias del Perú 2002-2012. Lima: Ministerio de Salud del Perú; 2002 [Accesado 15 enero 2003]. Disponible en: http://www.minsa.gob.pe/ocom/lineamientos/ Lineamientos7.pdf

22. Organización Panamericana de la Salud. Liderazgo en salud panamericana. Washington DC: Organización Panamericana de la Salud; 1998 (documento oficial N² 287).

23. Caminal J. La medida de la satisfacción: un instrumento de participación de la población en la mejora continua de la calidad de los servicios sanitarios. Rev Calidad Asistencial. 2001;16:276-9.

24. Carr-Hill R. The measurement of patient satisfaction. J Public Health Med. 1992;14(3):236-49.

25. Williams B. Patient satisfaction: a valid concept?. Soc Sci Med. 1994;38(4):509-16.

26. Grogan S, Conner M, Norman P, Willits D, Porter I. Validation of a questionnaire measuring patient satisfaction with general practitioner services. Qual Health Care. 2000;9:210-5. 
27. Duff L, Lamping D and Ahmed L. Evaluating satisfaction with maternity care in women from minority ethnic communities: development and validation of a Sylheti questionnaire. Int J Qual Health Care. 2001;13(3):216-30.

28. Caminal J, Sánchez E, Schiaffino A. El análisis por segmento de población insatisfecha: una propuesta para optimizar la información de las encuestas de satisfacción global. Rev Calidad Asistencial. 2002;17(1):4-10.

29. Steine S, Finset A, Laerum E. A new, brief questionnaire (PEQ) developed in primary health care for measuring patients' experience interaction, emotion and consultation outcome. Fam Pract. 2001;18(4):410-8.

30. Avis M, Bond M and Arthur A. Questioning patient satisfaction: an empirical investigation in two outpatient clinics. Soc Sci Med. 1997;44(1):85-92.

31. Delgado A, Lopez LA, Luna JD. Influencia del modelo organizativo en la satisfacción de los usuarios. Aten Primaria. 1995;16:314-21.

32. Lafond A. Improving the quality of investment in health: lessons on sustentability. Health Policy Plann. 1995;10:6376.

33. Hjortdahl P, Laerum E. Continuity of care in general practice: effect on patient satisfaction. BMJ. 1992; 304(6837):1287-90.

34. Hall J, Dornan M. What patients like about their medical care and low often they are asked: A meta-analysis of the satisfaction literature. Soc Sci Med. 1988;27: 935-9.

35. Seclen-Palacin J, Benavides B, Jacoby E, Velásquez A, Watanabe E. ¿Existe relación entre programas de mejora de calidad y satisfacción de usuarias de control prenatal?: a propósito de una experiencia. Rev Pan Salud Pública. 2004;16(3):149-57.

36. Carpio C, Choquepuma M, Vargas J, Basurco R, Arias M. Calidad de atención según el grado de satisfacción de las usuarias. En: Ministerio de Salud del Perú. Proyecto 2000: Investigaciones operativas en salud materno infantilCompendio-Perú. Lima: Pathfinder International, USAID; 2002.

37. Habicht JP, Victora CG, Vaughan JP. Evaluation designs for adequacy, plausibility and probability of public health programme performance and impact. Int $\mathrm{J}$ Epidemiol. 1999;28:10-8.

38. Meakin R, Weinman J. The'Medical Interview Satisfaction Scale' (MISS 21) adapted for British general practice. Fam Pract. 2002;19(3):257-3.

39. Parasumaran A, Zeithaml V, Berry L. SERVQUAL: a multiple item scale for measuring consumer perceptions of service quality. Journal of Retailing. 1998;64(1):12-37.

40. Cerna PN. Calidad del servicio expresada en la satisfacción del usuario externo e interno del centro de salud "Baños del Inca” [Disertación de maestría]. Lima: Facultad de Salud Pública, Universidad Peruana Cayetano Heredia; 2002.

41. Najera AP. Medición de la calidad del servicio en el centro de salud “Jesús". Red de salud Cajamarca II, 2000
[Disertación de maestría]. Lima: Facultad de Salud Pública, Universidad Peruana Cayetano Heredia; 2002.

42. Rojas AJ. Medición de la calidad del servicio en el centro de salud "Pachacútec" - Red de salud II Cajamarca - 2000 [Disertación de maestría]. Lima: Facultad de Salud Pública, Universidad Peruana Cayetano Heredia; 2002.

43. Aguirre SO. Evaluación de la calidad del servicio en el centro de salud de Otuzco [Disertación de maestría]. Lima: Facultad de Salud Pública, Universidad Peruana Cayetano Heredia; 2002.

44. Chilon ME. Medición de la calidad de los servicios en el puesto de salud "Simón Bolívar" - Red de salud II Cajamarca - 2000 [Disertación de maestría]. Lima: Facultad de Salud Pública, Universidad Peruana Cayetano Heredia; 2002.

45. Apaza PR, Evaluación de la calidad del servicio en el módulo de atención integral del adulto mayor. Hospital Marino Molina Scappe, 2002 [Disertación de maestría]. Lima: Facultad de Salud Pública, Universidad Peruana Cayetano Heredia; 2003.

46. Fiscella K, Franks P, Gold M, Clancy C. Inequality in quality: addressing socioeconomic, racial and ethnic disparities in health care. JAMA. 2000;283:2579-84.

47. Aaron KA, Clancy CM. Improving quality and reducing disparities: toward a common pathway. JAMA. 2003;289:1300-34.

48. Hall J, Dornan M. Patient sociodemographic characteristics as predictors of satisfaction with medical care: a metaanalysis. Soc Sci Med. 1990;30(7):811-8.

49. Rahmqvist M. Patient satisfaction in relation to age, health status and other background factors: a model for comparisons of care units. Int J Qual Health Care. 2001;13(5):385-90.

50. Organización Mundial de la Salud. Informe sobre la salud en el mundo: forjemos el futuro. Ginebra: OMS; 2003.

51. Hotchkiss D. Expansion of rural health care and the use of maternal services in Nepal. Health Place. 2001;7(1):39-45.

52. Bower P, Roland M, Campbell J, Nicola M. Setting standards based on patients' views on access and continuity: secondary analysis of data from the general practice assessment survey. Br Med J. 2003;326:258- 62 .

53. Qatari GA, Haran D. Determinants of users' satisfaction with primary health care settings and services in Saudi Arabia. Int J Qual Health Care. 1999;11(6):523-31.

Manuscrito recibido el 30 de marzo de 2005 y aceptado para publicación el 05 junio de 2005.

Correspondencia: Dr. Juan A. Seclén-Palacin

Facultad de Salud Pública y Administración,

Universidad Peruana Cayetano Heredia

Av. Honorio Delgado 430, Urb. Ingeniería, S.M.P.

Lima 31, Perú.

Correo-e: jseclen@yahoo.com 